\title{
Target Design for XUV Probing of Radiative Shock Experiments
}

\section{U. Chaulagain, C. Stehlé, P. Barroso, M. Kozlova, J. Nejdl, F. Suzuki Vidal and J. Larour}

Journal of Nepal Physical Society

Volume 6, Issue 1, June 2020

ISSN: 2392-473X (Print), 2738-9537 (Online)

\section{Editors:}

Dr. Binod Adhikari

Dr. Manoj Kumar Yadav

Mr. Kiran Pudasainee

JNPS, 6 (1), 30-41 (2020)

DOI: http://doi.org/10.3126/jnphyssoc.v6i1.30514

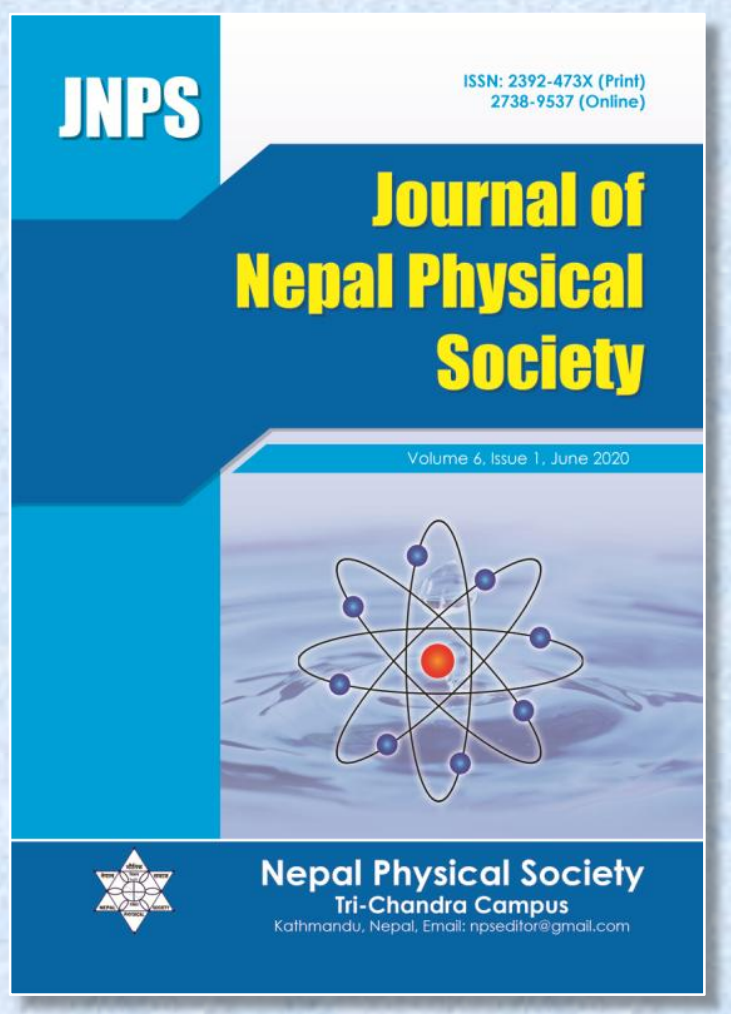

Published by:

Nepal Physical Society

P.O. Box: 2934

Tri-Chandra Campus

Kathmandu, Nepal

Email: npseditor@gmail.com 


\title{
Target Design for XUV Probing of Radiative Shock Experiments
}

\author{
U. Chaulagain ${ }^{1,}{ }^{*}$, C. Stehlé 2 , P. Barroso ${ }^{3}$, M. Kozlovaa ${ }^{1,4}$, J. Nejdl ${ }^{1}$, F. Suzuki Vidal ${ }^{5}$ and J. Larour $^{6}$ \\ ${ }^{1}$ ELI Beamlines, Institute of Physics ASCR, Na Slovance 1999/2, Prague, 182 21, Czech Republic \\ ${ }^{2}$ LERMA UMR 8112, Observatoire de Paris, PSL Research University, UPMC, Sorbonne University, CNRS, France \\ ${ }^{3}$ GEPI, UMR 8111, Observatoire de Paris, PSL Research University \\ ${ }^{4}$ Institute of Plasma Physics ASCR, Za Slovankou 1782/3, Prague, 182 00, Czech Republic \\ ${ }^{5}$ The Blackett Laboratory, Imperial College, Prince Consort Road, London SW7 2AZ, UK \\ ${ }^{6}$ LPP, UMR 7648, Ecole Polytechnique, UPMC, Sorbonne Université, U-PSud, 91128 Palaiseau, France \\ *Corresponding Email: uddhab.chaulagain@eli-beams.eu
}

Received: 12 Apr., 2020; Revised: 20 May, 2020; Accepted: 28 Jun., 2020

\begin{abstract}
Radiative shocks are strong shocks characterized by plasma at a high temperature emitting an important fraction of its energy as radiation. Radiative shocks are commonly found in many astrophysical systems and are templates of radiative hydrodynamic flows, which can be studied experimentally using high-power lasers. This is not only important in the context of laboratory astrophysics but also to benchmark numerical studies. We present details on the design of experiments on radiative shocks in xenon gas performed at the $\mathrm{kJ}$ scale PALS laser facility. It includes technical specifications for the tube targets design and numerical studies with the 1-D radiative hydrodynamics code MULTI. Emphasis is given to the technical feasibility of an XUV imaging diagnostic with a $21 \mathrm{~nm}(\sim 58 \mathrm{eV})$ probing beam, which allows to probe simultaneously the post-shock and the precursor region ahead of the shock. The novel design of the target together with the improved X-ray optics and XUV source allow to show both the dense post-shock structure and the precursor of the radiative shock.
\end{abstract}

Keywords: Radiative hydrodynamics, Radiative shocks, micro targets, Laboratory astrophysics, X-ray laser, XUV probing.

\section{INTRODUCTION}

Radiative shocks are found in many astrophysical scenarios, e.g. during the stellar breakthrough of a supernova shock [1], in accretion shocks [2, 3], interaction of supernova remnants with the interstellar medium (ISM) [4, 5], and in the stellar jets from young stars $[6,7]$. All these shocks are characterized by high Mach numbers $(\mathrm{M}>>1)$. For instance, in accretion shocks in young stars, matter is channelled from the protostellar disk towards the photosphere at $\sim 400 \mathrm{kms}^{-1}$ leading to an accretion shock and a subsequent reverse shock propagating backwards. This leads to strong heating (up to several $10^{6} \mathrm{~K}$ ) and strong X-ray emission [8-13]. In general, the angular resolution of present astronomical observations is not sufficient to resolve the structure of these shocks, and thus their fine structure and dynamical behaviour remains poorly constrained.

Understanding the properties of radiative shocks is a fundamental problem since they are the basis for the interpretation of observations of various astrophysical phenomena. At high shock velocities, a significant fraction of the energy in the shock is converted to radiation. Depending on the density of the upstream medium, radiation can escape freely or be re-absorbed in the medium where the shock propagates, ionizing it and forming a 'radiative precursor'. This process is usually accompanied by strong post-shock cooling [14, 15].

High-power lasers and pulsed power Z-pinches are capable of creating high energy density 
environments allowing to study a wide variety of states of matter of astrophysical interest over many orders of magnitude in density, pressure and temperature [16]. Several radiative shock experiments have been performed in different laboratories in the last decades, aiming at understanding the coupling of radiation with hydrodynamics and the role of radiation in the shock structure [17-28]. Measurements of these extreme plasma conditions are critical for theoretical modelling and to validate numerical simulations. Although the conditions for shock generation and characteristic plasma parameters between the laboratory and space can be significantly different.

There are three dimensionless numbers; Mach number (M), Boltzmann number (Bo) and Mihalas number (R), are commonly used to characterized the measure the strength of the shock and the effect of the radiation in the shock. The strength of a shock is defined Mach number (M), which is the ratio of the shock velocity to the local sound speed. The local sound speed can be taken either with the initial gas condition or upstream gas conditions (conditions of Radiative Precursor). Bo and $\mathrm{R}$ determines the extents of radiation that plays the role in shock dynamics. Bo compares the local material energy flux to the radiative flux and $\mathrm{R}$ compares the internal energy density with radiation density. If $\mathrm{Bo} \leq 1$, the flow is in the radiation flux dominated regime. The radiative shock we reported is a radiation flux dominated regime shock wave with the value of Bo is $\sim 0.01$. Some of the recent experiments have demonstrated the ability to obtain dimensionless Mach and Boltzmann number of the same order of magnitude and thus both phenomena are in conditions where radiative flux plays an important role see e.g. [21].

However, it is important to note that the coupling (e.g. absorption) of radiation with hydrodynamics is more important in the laboratory than in astrophysics (where the medium is relatively dilute) making one to one comparison challenging. In the particular case of stellar accretion shocks [11], e.g. at the interface of an accretion column with the photosphere of a star, previous experiments (see e.g. [20-28] and references therein) have looked at narrowing the gap between astrophysics and the laboratory by producing shocks with velocities of $\sim 10-100 \mathrm{~s} \mathrm{kms}^{-1}$, i.e. upstream Mach numbers $\sim 100$ (with respect to cold gas) and Boltzman numbers $\sim 10^{-2}-10^{-3}$.
Although radiative shocks have been extensively studied in the laboratory, the majority of previous experiments have focused either on the study of the radiative precursor using optical laser probing [17, $18]$, or the post shock structure with hard X-ray backlighting [21, 24]. Although optical probing is often used to characterize laser-produced plasmas, it is limited to moderate electron densities due to strong absorption of the probing laser beam near the critical density (i.e. $6.4 \times 10^{20} \mathrm{~cm}^{-3}$ at 1.3 $\mu \mathrm{m})$. As a consequence, this diagnostic is unable to probe the dense shock front, the post-shock and the radiative precursor region very close to the shock front. Hard X-ray backlighting, on the other hand, is almost transparent to the typical conditions of a radiative precursor (i.e. very small changes in mass density) and to the best of our knowledge, so far, no experiment has been able to provide images of the entire shock structure (i.e. precursor, shock front and post-shock). Due to its moderate absorption in the conditions of the post shock and of the precursor, soft X-ray/XUV probing is an ideal tool to probe for this purpose.

Alternatively, by using ultrashort X-ray sources betatron or Compton [29, 30], based on the Laser Wakefield acceleration (LWFA) [31], it would be possible to resolve the dynamics of fast-moving shock front. The intrinsic temporal resolution of this X-ray source is less than $10 \mathrm{fs}$. In addition, betatron source have a very small source size $(\sim \mu \mathrm{m})$, with this feature, it is possible to get the phase contrast image $[32,33]$ which provides both absorption and phase information. To drive such Xray source a very high peak power laser system required. However, at the moment these sources are not available in large energy laser facilities.

In this paper, we present details of the design and results of radiative shock experiments performed at the PALS laser facility. We used the PALS $10 \mathrm{~mJ}$ $\mathrm{Zn}$ XUV laser $(\lambda=21.2 \mathrm{~nm}$ equivalent to a photon energy of $\sim 58 \mathrm{eV}$ with a pulse duration of $\mathrm{l}=150$ ps) [[19],[34]] for instantaneous imaging of a radiative shock propagating at a velocity of $\sim 50$ $\mathrm{kms}^{-1}$ in a rectangular shock tube filled with lowdensity xenon $\left(\sim 1.5 \mathrm{mgcm}^{-3}\right.$ equivalent to $\sim 0.3$ bar). This is a technically challenging diagnostic as, despite being one of the most energetic XUV lasers in the world, it requires very high-quality optics to minimize any beam losses. Thus, key elements in the experimental design were the use of a half cavity for the laser, an XUV multilayer spherical mirror for imaging, and appropriate target windows that could withstand the gas pressure 
inside the targets while allowing for sufficient XUV transmission. Moreover, the generation of the X-ray laser at PALS requires $\sim 1 \mathrm{~kJ}$ of IR laser beam. As a result, the remaining energy to drive the shock was relatively small, $(\sim 60 \mathrm{~J})$, which imposed a careful optimization of the target.

\section{SHOCK TUBE TARGETS}

\subsection{Piston design and optimization}

The strength of radiative effects on a shock driven in a gas depends mainly on two parameters: the shock velocity and the initial gas density. To generate a radiative shock, a high-power laser is focused onto a solid foil that acts as an ablator by converting the laser energy into mechanical momentum, i.e. behaving like a piston pushing and compressing the gas. Initially, during the ablation process a shock is generated which propagates through the foil and then breaks out into the gas. For a given laser intensity and within a given range of gas pressures, the shock velocity in the gas is mainly determined by the material and the thickness of the piston. To optimize the shock velocity, the thickness of the piston must be large enough so that the rear surface of the piston remains in its solid phase after laser irradiation. If the piston is too thin, the laser easily ablates it leading to insufficient mass to act as a piston. On the other hand, if the piston is too thick, the remaining mass is too large and the piston velocity is reduced, leading to slower shock velocities and reduced radiative effects. Thus, a careful selection of piston materials and thicknesses is crucial for the design of radiative shocks experiments.

The goal is to optimize this piston in order to get the highest velocity in xenon while limiting the xenon preheating by the ablated piston material. For the experiments presented here, the piston was made of a layer of polystyrene (hereafter $\mathrm{CH}$ ) followed by a thin layer of gold in contact with the gas filling the target (xenon). This gold layer acts as a radiation shield preventing any pre-heating of the xenon and the walls of the gascell by X-rays generated during the $\mathrm{CH}$ ablation. The gold thickness should be thick enough in order to block the $\mathrm{keV}$ emission from the ablated $\mathrm{CH}$ but not too much in order not to slow down the shock. A gold layer with a thickness of 0.5 microns was used due to its transmission of $\sim 1 \%$ at a photon energy of $1 \mathrm{keV}$.

In order to achieve the suitable intensity required to launch a radiative shock (a few $10^{14} \mathrm{Wcm}^{-2}$ ), a spot size of $\sim 350 \mu \mathrm{m}$ diameter is required. For the experiments to be in a regime where it is possible to produce a radiative precursor having moderate strong gas-absorption for XUV probing, the gas pressure $\mathrm{P}$ should be between $\sim 0.1$ bar and 0.5 bar. Thus, for the present experiments the xenon pressure was set to 0.3 bar (i.e. an initial mass density of $1.5 \times 10^{-3} \mathrm{gcm}^{-3}$ ).
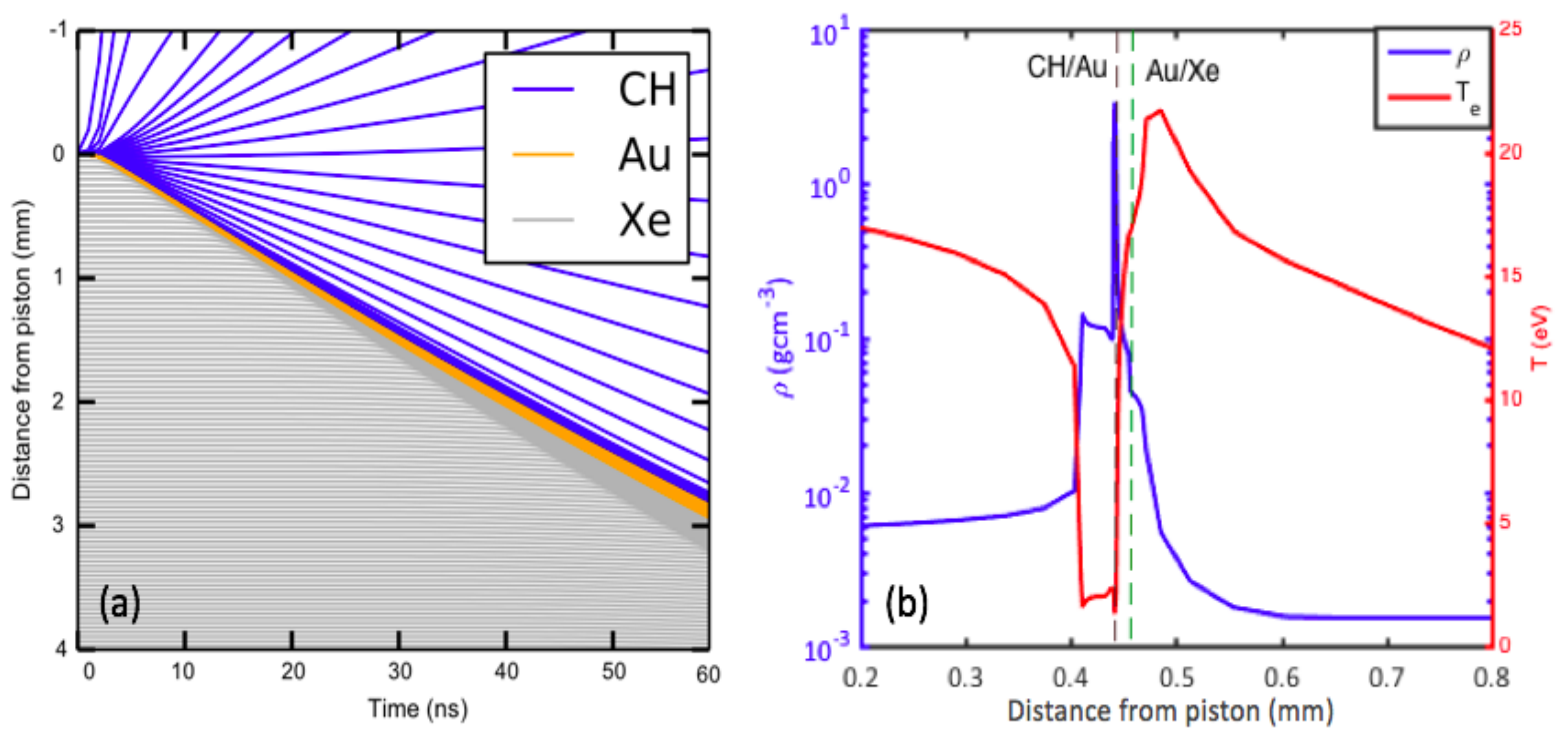

Fig. 1: MULTI simulations in the case of a 10- $\mu \mathrm{m}$ piston made of $\mathrm{CH}$ followed by $0.5 \mu \mathrm{m}$ Au layer and $6 \mathrm{~mm}$ of xenon gas at 0.3 bar. (a) Positions of the different layers versus time. The laser is incident from the top at $t=0$ ns. (b) Mass density and electron temperature profiles as a function of distance from the piston at 10 ns after the arrival of the driving laser pulse. The laser is incident from the left and the zero position is the initial position of the piston. 
To optimize the design of the piston, the 1-D Lagrangian radiation hydrodynamics code MULTI [42] was used. The code solves the onedimensional planar hydrodynamic equations coupled to the radiation transfer equations using a fully implicit numerical scheme. It uses tabulated equation of state (EoS) data, Planck and Rosseland opacities that are generated externally using a grey approximation. Thermal conduction is described within a flux-limited electron conduction model based on Spitzer's formula. The deposition of the laser is computed with an inverse-bremsstrahlung model. The simulation setup consisted of three layers: $10 \mu \mathrm{m} \mathrm{CH}, 0.5$ $\mu \mathrm{m} \mathrm{Au}, 6 \mathrm{~mm}$ of xenon at a 0.3 bar pressure and the laser parameters: $\lambda=1315 \mathrm{~nm}$, a pulse duration of $350 \mathrm{ps}$ and an energy of $60 \mathrm{~J}$ provides peak intensity of $1.3 \times 10^{14} \mathrm{Wcm}^{-2}$.

Fig 1a shows a position vs time plot of the different layer zones, with the $0 \mathrm{~ns}$ time marking the time when the driving laser hits the piston (incident from the top in the plot). The shock starts in xenon just after the end of the laser pulse. The $\mathrm{CH}$ layers move backwards due to ablation. The gold foil expands with time and the thickness of the post shock in xenon increases with time. From this figure, we can estimate the simulated velocity of the $\mathrm{Xe} / \mathrm{Au}$ interface at about $\sim 48 \mathrm{kms}^{-1}$. This velocity of the $\mathrm{Xe} / \mathrm{Au}$ interface remains almost constant for more than $50 \mathrm{~ns}$ after laser pulse irradiation. The gold layer expands with time from $0.5 \mu \mathrm{m}$ at $\mathrm{t}=0 \mathrm{~ns}$ to $30 \mu \mathrm{m}$ at $=20 \mathrm{~ns}$.

The variation of mass density $(\rho)$ and temperature (T) along the different material layers at $10 \mathrm{~ns}$ are reported in the Fig1b. From left to the right the profiles show a tail of dilute warm $(\sim 15 \mathrm{eV}) \mathrm{CH}$ layer at a density of $0.005 \mathrm{gcm}^{-3}$ (the initial density of $\mathrm{CH}$ was $1.1 \mathrm{gcm}^{-3}$ ), followed at $\sim 0.4$ $\mathrm{mm}$ by a dense shell of $\mathrm{CH}$ (up to $\sim 0.03 \mathrm{gcm}^{-3}$ ) at a low temperature of $\sim 2 \mathrm{eV}$. The gold layer is marked by a peak in mass density $\sim 3 \mathrm{gcm}^{-3}$ (initial density of $19 \mathrm{gcm}^{-3}$, expands to a thickness up to $20 \mu \mathrm{m}$. Finally, the peak in electron temperature marks the position of the shock wave in Xenon which is located at $\sim 0.48 \mathrm{~mm}$.

In order to characterise the dependence of piston thickness for a fixed laser energy, simulations were ran for different thickness ranging between 5 and $25 \mu \mathrm{m}$ of $\mathrm{CH}$. The simulations show that the $\mathrm{Au}-\mathrm{Xe}$ interface velocity for $25 \mu \mathrm{m} \mathrm{CH}$ is $\sim 30 \mathrm{kms}^{-1}$ and for $5 \mu \mathrm{m} \mathrm{CH}$ is $\sim 70 \mathrm{kms}^{-1}$ at $10 \mathrm{~ns}$. For 5 microns, the velocity is at maximum. However, a close inspection of the mass density, temperature conditions indicate that most of the $\mathrm{CH}$ layer is ablated up to the gold layer, which in turn is heated more.

Thus 5 microns is the minimum thickness acceptable for the piston. In addition, thinner pistons are prone to technical difficulties related to target manufacture feasibility and high costs. Most importantly, thin pistons cannot withstand the pressure difference of 1 bar required during the gas filling process. Bearing these factors in mind and with the laser energy accessible to drive the shock at PALS, a $10 \mu \mathrm{m}$ thick $\mathrm{CH}$ layer was chosen as the piston for the experiments. These 1D simulations were performed in order to optimize the thickness of the piston but not to compare the experiment. The experimental results were simulated using 2D Radiative Hydrodynamics code with the state-of-the-art opacities and the measured focal spot [20, 41] and laser energy reported in [46].

\subsection{Shock tube design}

Laser generated radiative shock targets can be divided broadly into two classes: shock tubes in which their internal cross section is adapted to the diameter of the shock wave, or cells with larger cross sections (see ref. [23, 24, 34, 37). In the first case, the underlying idea is to produce a planar shock (i.e. with a 1-D geometry), facilitating the comparison with simulations and models. However, previous studies have shown that the radiation emitted by the shock can be absorbed by the lateral windows on the targets with losses due to, e.g. albedo effects, thus leading to a curved shock geometry instead of a planar one. In the second case, the large cell volume allows for an unimpeded propagation of the shock, leading to a spherical shock geometry (e.g. see ref. [27, 28, 38, 40].

The targets in the experiments presented here are of the first type (shock tube), with a cross-section of $400 \times 400 \mu \mathrm{m}^{2}$ and a length of $6 \mathrm{~mm}$ which, for a typical shock velocity of $50 \mathrm{kms}^{-1}$, allows to probe the shock up to $\sim 100 \mathrm{~ns}$. The feasibility of the target design was aided by INGESYM Engineering and the bulk of the targets (made of aluminium) were micro machined and then assembled at the Pole Instrumental de l'Observatoire de Paris. A customised target holder was designed to allow for repeatable target positioning and centering during each experiment with a precision within $\sim 50 \mu \mathrm{m}$. The details of the target and its components are shown in Fig. 2. 
(a)

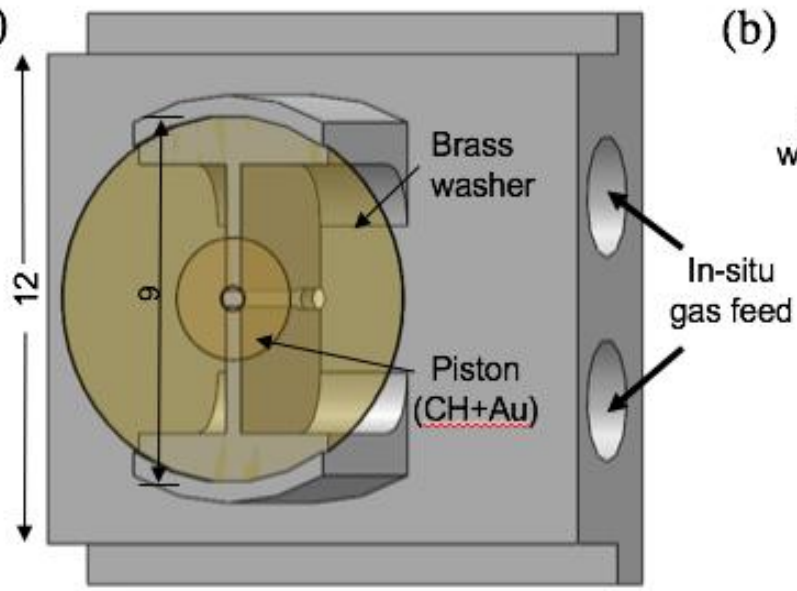

(b)

(c)

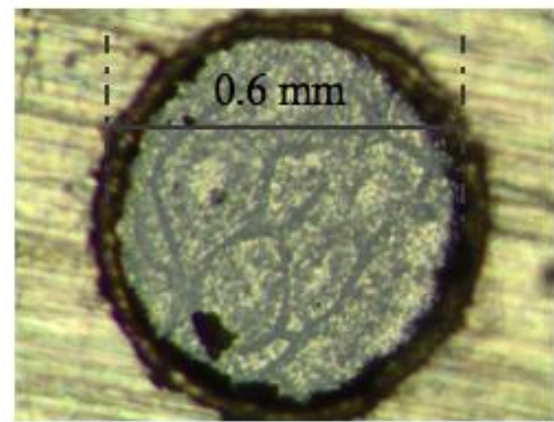

(d)

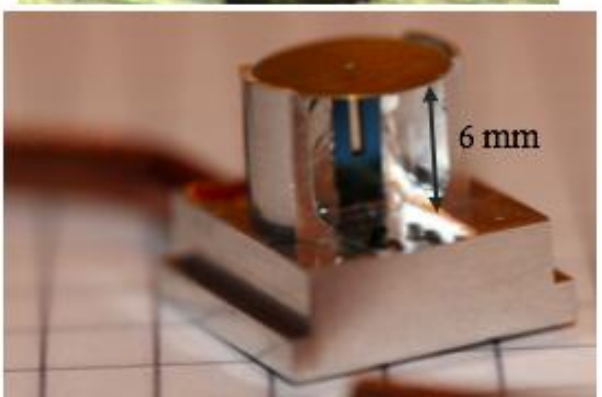

(e)

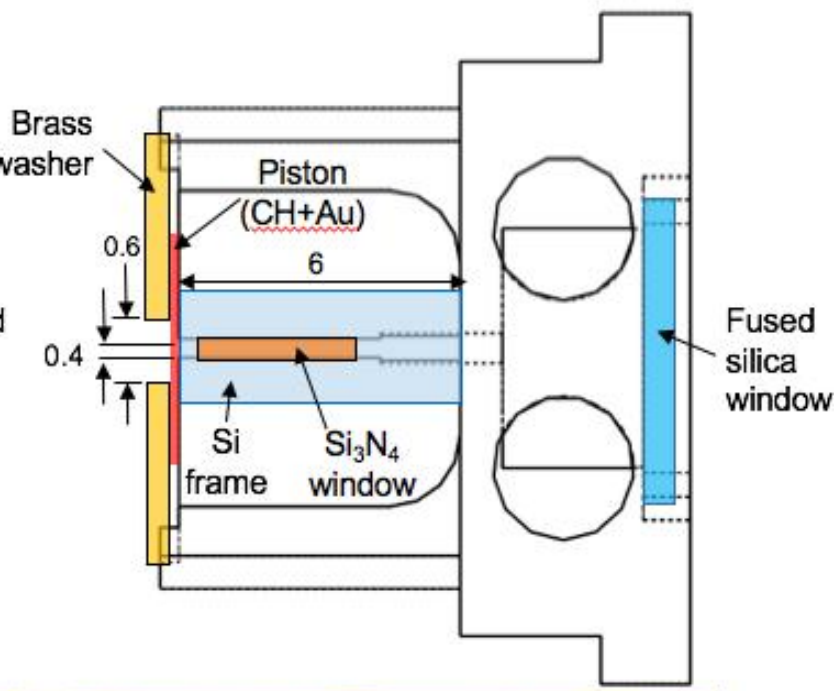

Fig. 2 Target assembly: ( $a$ ) and (b) show front and side-on CAD views of the target, with the circular brass washer holding the $\mathrm{CH}$ piston shown schematically. (c) $\mathrm{CH} / \mathrm{Au}$ piston seen from the side of polystyrene through the brass support (hole diameter $600 \mu \mathrm{m})$. (d) is the picture of the target and (e) target holder with a target and a high-speed IRD Silicon diode diagnostics.

As previously mentioned, the shock tubes in the targets are closed at one end by a piston made of a $10 \mu \mathrm{m}$ thick polystyrene $(\mathrm{CH})$ foil coated with a 0.5 $\mu \mathrm{m}$ gold layer. The gold was deposited by thermal vacuum evaporation (Joule effect) with a precision estimated to $0.1 \mu \mathrm{m}$. The multilayer piston was glued to a 9-mm diameter, $0.1 \mathrm{~mm}$ thick brass washer with a $0.6 \mathrm{~mm}$ diameter aperture on it centre, shown in Figs. 2a-b. The brass disk was then glued to the aluminium target structure with a centering estimated to $\pm 100 \mu \mathrm{m}$.

Fig. 2 (c) shows a microscope view of the Polystyrene side of the piston through the $0.6 \mathrm{~mm}$ aperture of the brass washer. Some defects can be seen the $\mathrm{CH} / \mathrm{Au}$ foil (as striations with a width $<\sim 10 \mathrm{um})$ are not expected to influence the quality of the shock wave itself after the laser ablation process. However, it can't be guaranteed that they would not influence the development of instabilities when the shock wave generated by the interaction of the laser with $\mathrm{CH}$ propagates through the different layers.

The rear end of the tube was closed by a $500 \mu \mathrm{m}$ thick fused silica window for target alignment before each shot. The alignment was done with a CW DPSS laser (532 nm, $40 \mathrm{~mW}$ ) injected at this end through an optical fibre. Taking benefit of the tiny transmission of the piston and of a long 
exposure of a visible camera we were able to localise the channel and performed alignment adjustments. The target is filled inside the vacuum chamber with xenon at a pressure of $0.3 \pm 0.01$ bar through a rigid 1-mm copper capillary tube connected to the gas cell reservoir (see section 2.3).

(a)

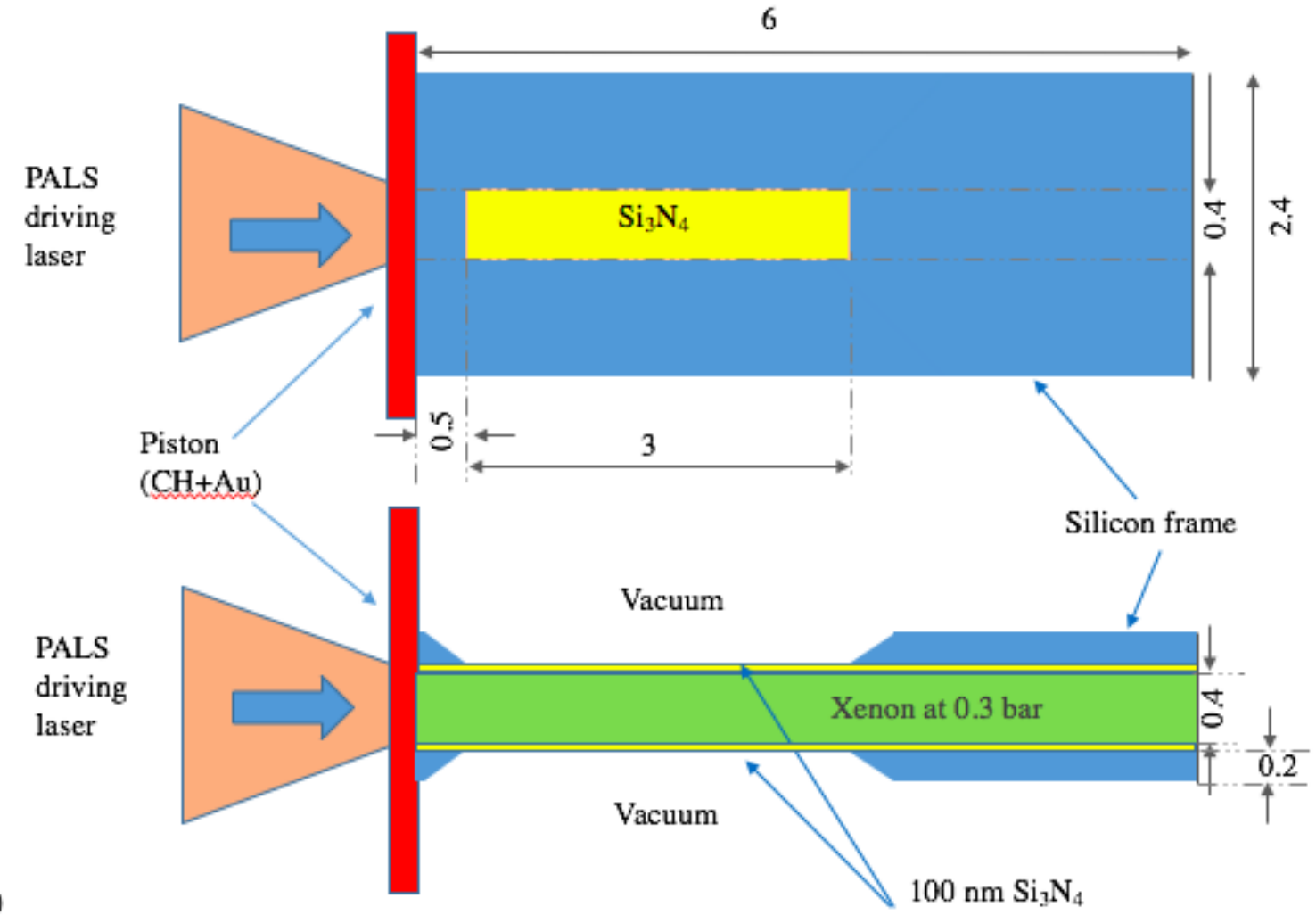

Fig. 3: Schematic view of the lateral windows used for XUV probing of a radiative shock. The windows consist of a Si3N4 membrane supported by a silicon frame. The shock tube was closed from two sides laterally by $100 \mathrm{~nm}$ silicon nitride windows (size $=3 \mathrm{~mm} \times 0.4 \mathrm{~mm}$ ) which are supported on a $200 \mu \mathrm{m}$ thick silicon frame $(2.4 \mathrm{~mm} \times 6 \mathrm{~mm})$, a) side-on view, b) End-on cut view.

In order to perform XUV probing of the plasma inside the targets, the lateral faces of the shock channel were enclosed by two $100 \mathrm{~nm}$ thick $\mathrm{Si}_{3} \mathrm{~N}_{4}$ windows $^{1}$ ( size $\left.=3 \mathrm{~mm} \times 0.4 \mathrm{~mm}\right)$ supported by a $200 \mu \mathrm{m}$ thick Silicon frame with a dimension of 2.4 $\mathrm{mm} \times 6 \mathrm{~mm}$ (see Fig. 3a and b)) and glued onto the Aluminium structure as shown as Fig. $2 \mathrm{~b}$ and $2 \mathrm{~d}$. These windows are adapted for XUV imaging as well as for time-and-space resolved plasma selfemission measurements using high-speed XUV

1 The maximum pressure difference which is allowed for a membrane of thickness $t$, section a is given by:

$\Delta P=2.44 \frac{t}{a} \sqrt{\frac{\sigma^{3}}{Y}}$ where $\sigma$ is breaking stress and $Y$ is the Young modulus. With $\sigma=2 \times 10^{9} \mathrm{Nm}^{-2}$, $Y=2.6 \times 10^{11} \mathrm{Nm}^{-2}, \mathrm{t}=100 \mathrm{~nm}$, and $a=400 \mu \mathrm{m}$ of $\mathrm{Si}_{3} \mathrm{~N}_{4}$ membrane, the pressure difference would be about 1 bar. diodes. The selection of these $\mathrm{Si}_{3} \mathrm{~N}_{4}$ windows was a critical step in the experimental design. The transmission of each of them at the probing wavelength $(\lambda=21.2 \mathrm{~nm})$ results in a net transmission through the 2 windows of $2 \%$, which means a dramatic loss of the XUV laser energy. The important point is that they are able to withstand the working difference of a difference of pressure of up to $\sim 0.3-0.7$ bar during the filling of the target inside the vacuum chamber before the experiments. These $\mathrm{Si}_{3} \mathrm{~N}_{4}$ membranes were manufactured by SILSON using hydroxide-based anisotropic etching of silicon wafers coated with LPCVD silicon nitride [45]. The cut-view schematic view of the silicon nitride window in Fig. $3 \mathrm{~b}$ shows the position of the silicon nitride membrane on the silicon frame. The silicon window was positioned in a way that the silicon 
nitride membrane was facing the gas-side on the target in order to avoid the membranes detaching from the frame due to the pressure difference of 0.3 bar against vacuum inside the chamber.

The targets were then fixed to a compact target holder (Fig. 2(e)) which also supported a high-speed XUV diode for plasma self-emission measurement diagnostics allowing to monitor the arrival of the shock in the field of view of the diodes, and consequently to derive the shock velocity. The precise design of the targets and target holder resulted in a reproducibility better than $\pm 50 \mu \mathrm{m}$ in the target positioning before the shot.

\subsection{Target filling and testing}

The thin, $100 \mathrm{~nm} \mathrm{Si}_{3} \mathrm{~N}_{4}$ windows are prone to break for an uncontrolled large difference in pressure between the internal and external part of the cell. To limit the risks of breaking, an in-situ filling mechanism was designed. There is no reservoir; the pipe is directly connected to the filling mechanism. The system includes a piezoresistive pressure transducer (Swagelok PTIS-AC.6-32), with a $10 \mathrm{k} \Omega$ bridge resistance working under a $14-30 \mathrm{~V}$ DC bias voltage. The theoretical response of the gauge was $0 \mathrm{~V}$ to $10 \mathrm{~V}$ for a range of absolute pressure: 0 to $1.6 \mathrm{bar}$, or relative pressure of -1 to +0.6 bar. The target and vacuum chamber were pumped simultaneously, which was then followed by a slow ( $\sim 1$ minute) gas filling of the target. Once the set pressure was achieved, all valves were closed to isolate the target from the gas bottle and prevent any leakage of xenon into the chamber. A leakage test of the filling system was performed using a test target, filled at atmospheric pressure, and placed inside the vacuum chamber. The filling system required about 20 seconds to stabilise. The system was connected through a $20-\mathrm{m}$ long coaxial cable to the control room allowing the remote reading of the pressure. A leakage test of the filling system was performed using a test target, filled at atmospheric pressure, and placed inside the vacuum chamber and observe the leakage, the leak rate was less than $5 \mathrm{mbar} / \mathrm{min}$. All targets that were shot during the experiments were pre-tested for leaks to the working pressure of $\sim 0.3$ bar.

\section{RESULTS AND DISCUSSION}

\subsection{PALS XUV Laser}

The XUV laser was generated by irradiating a 3cm-long high optically polished zinc slab by the
PALS iodine laser $(\lambda=1315 \mathrm{~nm})$ using a double sequence with a controllable delay $[23,34,39]$. A pre-pulse (a few $\mathbf{J}$ ) of the infra-red laser is used to produce a uniform pre-plasma, which is followed by a $500 \mathrm{~J}$ beam on target with a controlled delay of $10 \pm 0.5 \mathrm{~ns}$ after the pre-pulse (for more details see [43]). The XUV laser is characterized by a wavelength of $21.2 \mathrm{~nm}$, pulse duration of $150 \mathrm{ps}$, energy up to $4 \mathrm{~mJ}$ and peak spectral brightness of $10^{27}$ photons $\mathrm{s}^{-1} \mathrm{~mm}^{-2} \mathrm{mrad}^{-2}$. The beam has a divergence of $5.8 \times 3.8 \mathrm{mrad}^{2}$. The coherence properties and high brightness of this laser make it well-suited for XUV imaging of dense plasmas [39]. This laser is used here for side-on XUV characterisation of the radiative shock.

\subsection{XUV imaging}

As already mentioned, XUV probing of dense radiative shocks in gases requires a bright source but also with a uniform energy distribution. The energy distribution of the Zn PALS XUV laser is uniform over a several $\mathrm{mm}^{2}$ area [43]. For these experiments, and in addition to the $\mathrm{Si}_{3} \mathrm{~N}_{4}$ windows, a critical element is the imaging XUV mirror which must have a uniform and high reflexivity at the wavelength of the probing laser. For this purpose, we used a periodic multilayer one-inch spherical mirror, with a substrate made of fused silica substrate (roughness $0.2 \mathrm{~nm} \mathrm{rms}$, pitch polished at Institut d'Optique) and with a deposition layers consisting of 20 periods of $\mathrm{Al} / \mathrm{Mo} / \mathrm{B}_{4} \mathrm{C}$ with $\mathrm{B}_{4} \mathrm{C}$ capping layer, made at CEMOX platform in Orsay $[35,44]$. The reflectivity of this mirror is about $45 \%$ (at $21.2 \mathrm{~nm}$ ) with a focal length of $300 \mathrm{~mm}$.

During the experiments, the XUV laser is generated in a separate chamber from the target and reaches the xenon tube $20 \mathrm{~ns}$ after the driving beam (details of the experimental setup is reported in [20]). The laser beam passes side-on through the shock tube reaching the spherical multilayer mirror, which focuses the beam into an iron pinhole (diameter 500 $\mu \mathrm{m})$ located at the focal plane of the spherical mirror. This pinhole acts as a spatial filter, reducing the stray light from the target, and thus improving the contrast on the CCD detector over the strong plasma self-emission [39]. Since the size of the pinhole is very small compared to the focal length of the spherical mirror, the aberrations linked to the mirror are kept at a very low level. After passing through the pinhole, the laser beam reaches the cooled CCD camera filtered with a $0.4 \mu \mathrm{m}$ thick aluminium foil, which blocks the parasitic visible light coming from the chamber. The glass on the 
vacuum cooled CCD camera (MTE 2048, Princeton Instrument) was removed to allow XUV transmission. The XUV beam is very bright, including two $100 \mathrm{~nm} \mathrm{Si}_{3} \mathrm{~N}_{4}$ membranes and 0.4 $\mu \mathrm{m} \mathrm{Al} \mathrm{foil,} \mathrm{in} \mathrm{the} \mathrm{imaging} \mathrm{scheme,} \mathrm{we} \mathrm{have}$ received over 25000 counts on the X-ray CCD. The magnification of the imaging setup was measured by placing a Nickel grid (G2002N, grid pitch $=125 \mu \mathrm{m}$ from Agar Scientific Ltd) on a test target. The section of the grid viewed by the probing laser is shown in the (a-b). Taking the pitch of the grid of $125 \mu \mathrm{m}$, the magnification of the imaging setup is measured to be equal to 4.85 . The limiting spatial resolution of the imaging setup is about $22 \mu \mathrm{m}$ in the object coordinate.

A sample snapshot of XUV image of a radiative shock is reported in the Fig. 4 (c). The details explanation of this shock image and its analysis has been already published Ref [20]. In Fig. 4 (c), we have reported the XUV image of the radiative shock section viewed through the Si3N4 windows at $20 \mathrm{~ns}$ after shock the lunching of the shock. The

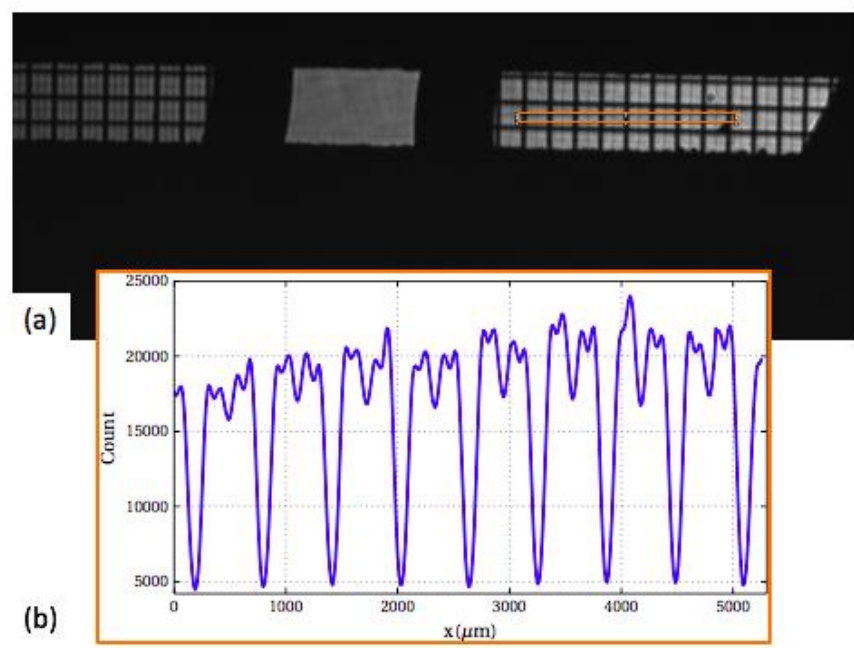

XRL image shows three different regimes of the radiative shock: the post-shock, shock front and the radiative precursor. The transmission at $21.2 \mathrm{~nm}$ along a longitudinal section of the image is along a line (yellow) at the position $y=250 \mu \mathrm{m}$ together with the transmission at $25 \mu \mathrm{m}$ below and above this line is reported in the Fig. 4d. A strong absorption of the probing laser by the piston residues ( $\mathrm{CH}$ and gold) is seen on the left end of the image with dark zone $(\mathrm{x}<680 \mu \mathrm{m})$ with a transmission of about $5-15 \%$. The position of shock front can be identified by rapid decrease of the transmission between 980 and $1020 \mu \mathrm{m}$ with the XRL transmission of value of $35 \%$. The shock is curved and elongated with its front located at about $1000 \mu \mathrm{m}$. The extension of radiative precursor is up to $1180 \mu \mathrm{m}$ with a transmission of up to $85 \%$. The thickness of the post-shock in the image is about 30 $\mu \mathrm{m}$. The position of shock front at $1000 \mu \mathrm{m}$, with a rapid drop in transmission due to strong absorption, which gives the shock front velocity is about 50 $\mathrm{kms}^{-1}$.

Fig. 4: (a) Magnification of imaging setup calculated by placing a grid a grid placed side-on on a test target. (b) Line out of the selected zone of the grid and the x-axis with the image coordinates (c) Crop of the section highlighting the shock feature. (d) transmission profiles along a horizontal section at the position of the yellow line on the top and 25 $\mu m$ above and below of it. The profiles are normalized to the maximum signal in the image which is at the right end of the image where the gas is unperturbed.

This result is consistent with the plasma selfemission measurement using the high-speed XUV diode reported in the Ref [20] and also supported by the 2D numerical simulations obtained with ARWEN code [46]. The second bump at $\sim 1070 \mu \mathrm{m}$ has been attributed to the behaviour of the xenon opacity at $21.2 \mathrm{~nm}$ for a given density and the temperature of the radiative precursor [48]. The state of art monochromatic opacities of xenon at the XUV probing wavelength for different temperature of shock region were calculated using the RAPCAL code [47], the resulted are reported in the Ref [20].

\section{CONCLUSIONS}

The novel design of the target together with the high energy Zn XUV laser at the PALS laser 
facility we were able to show, for the first time, an unambiguous and complete image of the pre- and post-shock regions in a radiative shock. These preliminary experiments open the door for future investigations of such hypersonic flows. Among possible improvements it is worth pointing out: 1) the transmission of the XUV target windows can be improved by using Silicon windows instead of $\mathrm{Si}_{3} \mathrm{~N}_{4}$ windows due to their increased transmission of $65 \%$ per window for a $150 \mathrm{~nm}$ thickness compared to a $13 \%$ transmission for 100 $\mathrm{nm} \mathrm{Si}_{3} \mathrm{~N}_{4}$. Preliminary tests of silicon windows with a thickness of $150 \mathrm{~nm}$ have been performed at PALS showing promising results. 2) The uniformity of the $\mathrm{CH} / \mathrm{Au}$ piston can be improved by using Parylene-N instead of polystyrene [26]. Further improvements in the XUV imaging mirrors and in the energy of PALS's XUV laser would allow to probe denser plasma conditions in a radiative shock by implementing e.g. XUV interferometry and temperature measurements with X-ray Thomson scattering. Current X-ray backlighting sources have large source sizes $(\sim 100$ $\mu \mathrm{m})$ and longer pulses which are not achieving the required resolution to characterize dense plasmas. However new ultrashort X-ray sources based on LWFA that can be scale from few $\mathrm{keV}$ to 10 's of $\mathrm{keV}$ [49], have been considered to be implemented in the large-scale laser system [50-52]. These sources can provide a high temporal resolution and high contrast image of fast-moving shock waves and enhance the diagnostics capability to study the dynamics of the dense plasmas.

\section{ACKNOWLEDGEMENTS}

The authors would like to thank Pascal Jagourel, Florent Reix, Thierry Melse from Pole Instrumental de l'Observatoire de Paris for their help in the target design. We acknowledge the support of the Scientific Council of Observatoire de Paris, the French CNRS/INSU Program PNPS, the Labex PLAS@PAR (ANR-11-IDEX-0004-02), the UFR de Physique of UPMC as also of EXTREME LIGHT INFRASTRUCTURE project no. CZ.1.05/1.1.00/02.0061,CZ.02.1.01/0.0/0.0/15_008 $/ 0000162$ and EC OP projects no. CZ.1.07/2.3.00/30.0057 and CZ.1.07/2.3.00/20. 0279, which were co-financed by the European Social Funds and the state budget of the Czech Republic and LASERLAB-EUROPE (grant agreement no. 284464, ECs Seventh Framework Programme). U.C, M. K. and J.N. would like to acknowledge the project Advanced research using high intensity laser produced photons and particles (CZ.02.1.01/0.0/0.0/16_019/0000789) from European Regional Development Fund (ADONIS). The results of the Project LQ1606 were obtained with the financial support of the Ministry of Education, Youth and Sports as part of targeted support from the National Programme of Sustainability II. We acknowledge project no. CZ.1.07/2.3.00/20.0279 that was co-financed by the European Social Fund and the state budget of the Czech Republic.

\section{REFERENCES}

[1] Ensman, L. and Burrows, A. Shock breakout in SN 1987A, The Astrophysical Journal, 393: 742755 (1992).

[2] Calvet, N. and Gullbring, E. The structure and emission of the accretion shock in t-tauri stars, The Astrophysical Journal, 509(2): 802 (1998).

[3] Argiroffi, C., Maggio, A., and Peres, G. X-ray emission from MP Muscae: an old classical $\mathrm{T}$ Tauri star, Astronomy \& Astrophysics, 465(1):L5-L8, (2007).

[4] Chevalier, R. A., Type ii supernovae SN 1987a and SN 1993j, Science 276.5317: 1374-1378 (1997)

[5] Nymark, T.K., Fransson, C., Kozma C., X-ray emission from radiative shocks in type ii supernovae Astronomy and Astrophysics, 449 171e192 (2006).

[6] Hartigan, P., Shock waves in outflows from young stars, In Jets in Young Stellar Objects (pages 111-122. Springer 2003).

[7] Reipurth, B. and Bally, J., Herbig-haro flows: probes of early stellar evolution, Ann. Rev. Astron, Astrophys 39 403-455 (2001).

[8] Donati, J. F., Gregory, S., Alencar, S., Hussain, G., Bouvier, J., Jardine, M., Ménard, F., Dougados, C., Romanova, M., and MaPP Collaboration, Magnetospheric accretion on the fully convective classical t tauri star dn tau, Monthly Notices of the Royal Astronomical Society 25;436(1):881-97 (2013).

[9] Hartmann, L., Calvet, N., Gullbring, E. and D'Alessio, P. Accretion and the evolution of $\mathrm{t}$ tauri disks, The Astrophysical Journal, 495(1):385 (1998).

[10] Orlando, S., Bonito, R., Argiroffi, C., Reale, F., Peres, G. Miceli, M., Matsakos, T., Stehlé, C., Ibgui, L., de Sa, L. and Chièze, J. P. Radiative accretion shocks along nonuniform stellar magnetic fields in classical $\mathrm{t}$ tauri stars, Astronomy \& Astrophysics, 559, A 127 (2013).

[11] Günther, H. M., Liefke, C., Schmitt, J. H. M. M., Robrade, J. and Ness, J. U. X-ray accretion 
signatures in the close CTTS binary V4046 Sagittarii, Astronomy \& Astrophysics, 459(2):L29-L32, (2006).

[12] Dupree, A. K., Brickhouse, N. S., Cranmer, S. R., Luna, G. J. M., Schneider, E. E., Bessell, M. S., Bonanos, A., Crause, L. A., Lawson, W. A., Mallik, S. V. and Schuler, S. C. TW Hya: spectral variability, X-rays, and accretion diagnostics, The Astrophysical Journal, 750(1):73 (2012).

[13] Sacco, G. G., Argiroffi, C., Orlando, S., Maggio, A., Peres, G., and Reale, F., X-ray emission from dense plasma in classical $T$ Tauri stars: hydrodynamic modeling of the accretion shock, The Astrophysical Journal, 491(2):L17-L20 (2008).

[14] Zel'dovich, I. B., Zel'dovich, Y. B. and Raizer, Y. P. Physics of shock waves and hightemperature hydrodynamic phenomena (Courier Dover Publications, 2002).

[15] Mihalas D. and Mihalas, B. W. Foundations of radiation hydrodynamics (Courier Dover Publications, 1999).

[16] Remington, B.A., Drake, R. P. and Ryutov, D. D. Experimental astrophysics with high power lasers and Z pinches, Reviews of Modern Physics, 78(3), 755 (2006).

[17] Fleury, X., Bouquet, S., Stehlé, C., Koenig, M., Batani, D., Benuzzi-Mounaix, A., Chieze, J.-P., Grandjouan, N., Grenier, J., Hall, T. and Henry, E. A laser experiment for studying radiative shocks in astrophysics, Laser and Particle Beams, 20(02):263-268 (2002).

[18] Stehlé, C., González, M., Kozlova, M., Rus, B., Mocek, T., Acef, O., Colombier, J.P., Lanz, T., Champion, N., Jakubczak, K. and Polan, J. Experimental study of radiative shocks at PALS facility" Laser and particle beams, 28(02):253261 (2010).

[19] Stehlé, C., Champion, N., Delattre, P.A., Chaulagain, U., Kozlová, M., Nejdl, J., Dostál, J., Krus, M., Larour, J., Suzuki-Vidal, F. and Acef, O. New diagnostics of laser generated shocks, EAS Publications Series, 58:155-159 (2012).

[20] Chaulagain, U., Stehlé, C., Larour, J., Kozlova, M., Suzuki-Vidal, F., Barroso, P., Cotelo, M., Velarde, P., Rodriguez, R., Gil, J.M. and Ciardi, A. Structure of a laser- driven radiative shock, High Energy Density Physics, 17: 106-113 (2015).

[21] Reighard, A.B., Drake, R.P., Dannenberg, K.K., Kremer, D.J., Grosskopf, M., Harding, E.C., Leibrandt, D.R., Glendinning, S.G., Perry, T.S., Remington, B.A. and Greenough, J. Observation of collapsing radiative shocks in laboratory experiments, Physics of Plasmas, 13(8):082901 (2006).
[22] Dizière, A., Michaut, C., Koenig, M., Gregory, C.D., Ravasio, A., Sakawa, Y., Kuramitsu, Y., Morita, T., Ide, T., Tanji, H. and Takabe, H. Highly radiative shock experiments driven by GEKKO XII, Astrophysics and Space Science 336, no. 1, 213-218 (2011).

[23] Stehlé, C., Kozlová, M., Larour, J., Nejdl, J., Champion, N., Barroso, P., Suzuki-Vidal, F., Acef, O., Delattre, P.A., Dostál, J. and Krus, M. New probing techniques of radiative shocks, Optics Communications, 285(1), 64-69 (2012).

[24] Chaulagain, U., Stehlé, C., Larour, J., Kozlová, M., Barroso, P., de Sá, L., Suzuki-Vidal, F., Acef, O., Auvray, P., Krus, M. and Dostal, J. "Laser experiment to study radiative shocks relevant to astrophysics." In SF2A-2013: Proceedings of the Annual meeting of the French Society of Astronomy and Astrophysics, pp. 213-217 (2013).

[25] Drake, R.P., Doss, F.W., McClarren, R.G., Adams, M.L., Amato, N., Bingham, D., Chou, C.C., DiStefano, C., Fidkowski, K., Fryxell, B. and Gombosi, T.I. Radiative effects in radiative shocks in shock tubes, High Energy Density Physics, 7 (3):130-140 (2011).

[26] Singh, R.L., Stehlé, C., Suzuki-Vidal, F., Kozlova, M., Larour, J., Chaulagain, U., Clayson, T., Rodriguez, R., Gil, J.M., Nejdl, J. and Krus, M., Experimental study of the interaction of two laser-driven radiative shocks at the PALS laser, High Energy Density Physics, 23, 20-30 (2017).

[27] Clayson, T., Suzuki-Vidal, F., Lebedev, S.V., Swadling, G.F, Stehle, C., Burdiak, G.C., Foster, J.M Skidmore, J., Graham, P., Gumbrell, E., Patankar, S., Spindloe, C., Chaulagain, U., Kozlova, M., Larour, J., Singh, R.L., Rodriguez, R., Gil, J.M., Espinosa, G. , Velarde, P. and Danson C. Counter-propagating radiative shock experiments on the Orion laser and the formation of radiative precursors, High Energy Density Physics, 23, 60-72 (2017).

[28] Suzuki-Vidal, F., Clayson, T., Stehlé, C., Swadling, G. F., Foster, J. M., Skidmore, J., Graham, P., Burdiak, G. C., Lebedev, S. V., Chaulagain, U., Singh, R. L., Gumbrell, E. T., Patankar, S., Spindloe, C., Larour, J., Kozlova, M., Rodriguez, R., Gil, J. M., Espinosa, G., Velarde, P., and Danson, C. Counterpropagating Radiative Shock Experiments on the Orion Laser, Physical Review Letters, 2;119(5):055001 (2017).

[29] Rousse, A., Phuoc, K.T., Shah, R., Pukhov, A., Lefebvre, E., Malka, V., Kiselev, S., Burgy, F., Rousseau, J.P., Umstadter, D. and Hulin, D. Production of a keV X-ray beam from synchrotron radiation in relativistic laser-plasma interaction, Physical review letters 93, no. 13: 135005 (2004).

[30] Kozlova, M., Andriyash, I., Gautier, J., Sebban, S., Smartsev, S., Jourdain, N., Chulagain, U., 
Azamoum, Y., Tafzi, A., Goddet, J. P., Oubrerie, K., Thaury, C., Rousse, A. and Ta Phuoc, K. Hard $X$ Rays from Laser-Wakefield Accelerators in Density Tailored Plasmas, Physical Review X 10, no. 1:011061 (2020).

[31] Tajima, T. and Dawson, J.M. Laser electron accelerator, Physical Review Letters, 43(4), p.267 (1979).

[32] Wood, J.C., Chapman, D.J., Poder, K., Lopes, N.C., Rutherford, M.E., White, T.G., Albert, F., Behm, K.T., Booth, N., Bryant, J.S.J. and Foster, P. S., Glenzer, S., Hill, E., Krushelnick, K., Najmudin, Z., Pollock, B. B., Rose, S., Schumaker, W., Scott, R. H. H., Sherlock, M., Thomas, A. G. R., Zhao, Z., Eakins, D. E. and Mangles, S. P. D. Ultrafast Imaging of Laser Driven Shock Waves using Betatron X-rays from a Laser Wakefield Accelerator, Scientific Reports 8, 11010 (2018).

[33] Chaulagain, U., Bohacek, K., Kozlova, M., Nejdl, J., Krus, M., Horny, V., Mahieu, B. and TaPhuoc, K. X-ray phase contrast imaging of biological samples using a betatron $\mathrm{X}$-ray source generated in a laser wakefield accelerator, In Laser Acceleration of Electrons, Protons, and Ions IV, vol. 10240, p. 1024014. International Society for Optics and Photonics, 2017.

[34] Kozlova, M., Nejdl, J., Krus, M., Prokupek, J., Dostal, J., Rus, B., Klisnick, A., Meng, L., Tissandier, F., Stehle, C. and Lefevre, R., Chaulagain, U., Champion, N., Barroso, P., Reix, F., Jagourel, P., Larour, J., Delmotte, F., Constancias, C., Suzuki-Vidal, F., Acef, O. Characterization of $\mathrm{Zn}$ X-ray laser at PALS centre, its applications in dense plasma probing and astrophysics. In X-Ray Lasers 2012 (151159). Springer, Cham (2014).

[35] Bauduin, D., Barroso, P., Melse, T., Croce, S., Gaudemard, J., Herpe, G. Réalisation de cellules pour des expériences de chocs radiatifs au LULI, LULI annual report 2015, 125 (2015).

[36] Stehle, C, Lefevre, R, Chaulagain, U. Chaulagain, U., Champion, N., Barroso, P., Reix, F., Jagourel, P., Larour, J., Meltchakov, E., Mercier, R. and Delmotte, F., Kozlova, M., Nejdl, J., Krus, M., Prokupek, J., Dostal, J., Constancias, C., SuzukiVidal, F., Acef, O. Critical Components for XUV Probing of Laser Driven Shocks." In X-Ray Lasers 2012, 239-242. Springer, (2014).

[37] Busquet, M., Barroso, P., Melse, T. and Bauduin, D. Miniature shock tube for laser driven shocks, Review of Scientific Instruments, 81(2), 023502 (2010).

[38] Spindloe, C., Wyatt, D., Astbury, S., Swadling, G. F., Clayson, T., Stehlé, C., Foster, J.M., Gumbrell, E., Charles, R., Danson, C. N., Brummitt, P., and Suzuki-Vidal, F. Design and fabrication of gas cell targets for laboratory astrophysics experiments on the Orion highpower laser facility, High Power Laser Science and Engineering, 5 (2017).

[39] Kozlová, M., Rus, B., Mocek, T., Polan, J., Homer, P., Snopek, D., Jakubczak, K., Fajardo, M. and Sardinha, A.B. High resolution X-ray laser backlighting of plasmas using spatial filtering technique, X-Ray Lasers 2008, 417-425 (2008)

[40] Koenig, M., Vinci, T., Benuzzi-Mounaix, A., Ozaki, N., Ravasio, A., Rabec le Glohaec, M., Boireau, L., Michaut, C., Bouquet, S., Atzeni, S. and Schiavi, A., Radiative shocks: An opportunity to study laboratory astrophysics, Physics of Plasmas, 13(5), 056504 (2006).

[41] Chaulagain, U., Stehlé, C., de Sá, L., Larour, J., Auvray, P., Kozlová, M., Krus, M., Dostal, J., Propupek, J., Suzuki-Vidal, F. and Barroso, P. Laboratory experiments of radiative shocks in the context of stellar accretion, In SF2A-2012: Proceedings of the Annual meeting of the French Society of Astronomy and Astrophysics, 305-307) (2012).

[42] Ramis, R., Schmalz, R. and Meyer-ter-Vehn, J. Multi - a computer code for one-dimensional multigroup radiation hydrodynamics, Computer Physics Communications, 49(3):475-505 (1988).

[43] Rus, B., Mocek, T., Präg, A.R., Kozlová, M., Jamelot, G., Carillon, A., Ros, D., Joyeux, D. and Phalippou, D., Multimillijoule, highly coherent Xray laser at $21 \mathrm{~nm}$ operating in deep saturation through double- pass amplification, Physical Review A, 66(6):063806, (2002).

[44] Meltchakov, E., Hecquet, C., Roulliay, M., De Rossi, S., Ménesguen, Y., Jérôme, A., Bridou, F., Varniere, F., Ravet-Krill, M.F. and Delmotte, F. Development of al-based multilayer optics for euv, Applied Physics A, 98(1):111-117, 2010.

[45] Michette, A.G. Morrison, G. R., and Buckley, C. J. X-ray Microscopy III, Vol. 67 of Springer Series in Optical Sciences (1992).

[46] Cotelo, M., Velarde, P., de la Varga, A.G., Portillo, D., Stehlé, C., Chaulagain, U., Kozlova, M., Larour, J. and Suzuki-Vidal, F. Simulation of radiative shock waves in $\mathrm{Xe}$ of last PALS experiments, High Energy Density Physics 17: 68-73 (2015).

[47] Rodriguez, R., Florido, R., Gil, J.M., Rubiano, J.G., Martel, P. and Mínguez, E. RAPCAL code: A flexible package to compute radiative properties for optically thin and thick low and high- $Z$ plasmas in a wide range of density and temperature, Laser and Particle Beams 26(3):433-48 (2008).

[48] Visco, A.J., Drake, R.P., Glenzer, S.H., Döppner, T., Gregori, G., Froula, D.H. and Grosskopf, 
M.J., Measurement of radiative shock properties by $x$-ray Thomson scattering, Physical review letters 108, no. 14:145001 (2012).

[49] Fourmaux, S., Hallin, E., Chaulagain, U., Weber, S. and Kieffer, J.C. Laser-based synchrotron Xray radiation experimental scaling, Optics Express 28, no. 3: 3147-3158 (2020).

[50] Chaulagain, U., Boháček, K., Vančura, J., Lamač, M., Yan, W., Gu, Y., Kozlová, M., TaPhuoc, K., Weber, S.A. and Nejdl, LWFADriven Betatron Source for Plasma Physics Platform at ELI Beamlines, In International Conference on X-ray Lasers, 117-123. Springer, Cham (2018).
[51] Nejdl, J., Mai, D.D., Chaulagain, U., Hort, O., Finke, O., Albrecht, M., Jurkovič, M., Lera, R., Karatodorov, S., Lamač, M. and Vančura, J. Progress on laser-driven $\mathrm{X}$-ray sources at ELI Beamlines, In X-Ray Lasers and Coherent X-Ray Sources: Development and Applications XIII (Vol. 11111, p. 111110I). International Society for Optics and Photonics (2019).

[52] Jourdain, N., Chaulagain, U., Kramer, D., Kumar, D., Majerovà, I., Schaumann, G., T. Tikhonchuk, V., Korn, G., Weber, S. The L4n laser beamline of the P3-installation: towards high-repetition rate high-energy density physics at ELI-Beamlines (submitted to Matter and Radiation at Extremes). 